\title{
Comparison of Efficiency of TACE plus HIFU and TACE alone on Patients with Primary Liver Cancer
}

\author{
Yuju Luo ${ }^{1}$ and Yu Jiang2
}

\begin{abstract}
Objective: To compare efficiency of trans-arterial chemo-embolization (TACE) plus high intensity focused ultrasound (HIFU), and TACE alone on patients with primary liver cancer.

Study Design: A descriptive, analytical study.

Place and Duration of Study: Department of Ultrasound, Daqing Oilfield General Hospital, China, from March 2015 to March 2017.

Methodology: A total of 90 primary liver cancer patients were randomly divided into control group and observation group, 45 cases in each group. Control group was treated with TACE alone, while observation group was treated with HIFU plus TACE. Alpha-fetoprotein (AFP), alanine amino-transferase (ALT), aspartate amino-transferase (AST), total bilirubin (TBIL), and frequency of complications, were compared in the two groups.

Results: The total remission rate of observation group was higher than that of control group $(p=0.017)$. At 6 months after treatment, AFP level in observation group was lower than that in control group $(p<0.001)$. There was no statistical difference in liver function indicators of ALT, AST, and TBIL between two groups ( $p=0.968,0.944$ and 0.973 , respectively). The incidence of digestive tract hemorrhage was lower than that in control group $(p=0.049)$. After one year of follow-up, the tumor recurrence rate and tumor metastasis rate in observation group were lower than that of control group $(p=0.036$ and 0.044 , respectively).

Conclusion: Treatment of primary liver cancer by TACE plus HIFU has a higher overall remission rate, causes little damage to normal liver tissue, can fully kill tumor cells and reduce postoperative local recurrence and metastasis rate with less adverse reactions.
\end{abstract}

Key Words: Primary liver cancer, TACE, HIFU, Liver function, Efficacy.

\section{INTRODUCTION}

Primary liver cancer refers to malignant tumor that occurs in liver cells or intrahepatic cholangiocarcinoma. ${ }^{1}$ As a common clinically refractory malignant tumor, it has a high incidence worldwide.2,3 Surgery, radiotherapy, chemotherapy, hormonal therapy are current popular cancer treatment options. These treatments are often associated with some inherent limitations. For instance, the anticancer drugs used for chemotherapy can quickly spread throughout the body and is ineffective in killing metastatic cancer cells; Trans-Arterial Chemo-Embolization (TACE) is an important tumor surgery. However, it is not effective in mitigating metastases, and it is still residual cancer in the tumor lesions. ${ }^{4}$ As a bloodless scalpel, high-intensity focused ultrasound (HIFU) is regarded as one of the most promising and representative noninvasive therapeutic modalities for cancer surgery. $5 \mathrm{lt}$ must be said, though, that HIFU are still in their infancy

Department of Ultrasound ${ }^{1}$ / Bone Surgery², Daqing Oilfield General Hospital, Daqing, Heilongjiang Province, 163001, China

Correspondence: Yuju Luo, Department of Ultrasound, Daqing Oilfield General Hospital, Daqing, Heilongjiang

Province, 163001 China

E-mail:bk79qvcq@sina.com

Received: April 24, 2018; Accepted: January 04, 2019 because of critical efficiency issues which remain to be solved. It is pointed out in some researches that HIFU seems to add clear survival advantages over TACE alone. 6 But some researchers believe HIFU have synergistic effect with TACE. ${ }^{7}$

The objective of this study was to compare the therapeutic efficiency of TACE plus HIFU and TACE alone on patients with primary liver cancer.

\section{METHODOLOGY}

This study was conducted at the Department of Ultrasound, Daqing Oilfield General Hospital, China, from March 2015 to March 2017. Approval was taken from the Hospital's Institutional Review Board. A total of 90 primary liver cancer patients were selected as research subjects. Inclusion criteria were in line with the diagnostic criteria of middle and advanced liver cancer, diagnosed as liver cancer by pathology, impossibility in radical resection, no combined distant metastasis; stable vital signs, normal coagulation mechanism, normal liver and kidney functions, complete clinical data, completing one year's postoperative follow-up. Exclusion criteria included, with combined severe mental illness, severe heart, lung, kidney disease, accompanied with other malignancies and hematological diseases, pregnant and lactating women with abnormal coagulation mechanisms, with possibility of radical resection, poor compliance and 
failure to complete follow-up. The patients were randomly divided into control group and observation group, with 45 cases in each group.

The control group was treated with TACE alone. Seldinger technique was employed to puncture the intubation tube, followed by celiac artery and hepatic artery angiography. The blood supply and branch status were observed. Later, portal vein and reflux were observed by superior mesenteric artery angiography. The catheter was then delivered to the left or right hepatic artery. The chemotherapeutic agents and lipiodol were injected into the tumor supply blood vessels. The chemotherapy drugs included 5 -fluorouracil $1.0 \mathrm{~g}$ and epirubicin $30 \mathrm{mg}$ (10 mg of which was mixed with an appropriate amount of ultra-liquid iodized oil to form emulsion). The dose of lipiodol emulsion varied depending on the size of the lesion and intraoperative tolerability of the patient, with an average dose at 10.5 $\mathrm{ml}$. TACE treatment was performed 1-2 times depending on the patient's tolerability, with intervals of 3-4 weeks.

The observation group was treated with HIFU plus TACE; HIFU was performed 2-4 weeks after TACE treatment. The parameters of HIFU tumor treatment system were: frequency $0.8 \mathrm{MHz}$, focal length $150 \mathrm{~mm}$ and treatment duration $4946-16223 \mathrm{~s}$. The therapeutic range and therapeutic dose were adjusted by monitoring B-mode ultrasound images during the treatment (Figure 1). Forty-five patients were treated with HIFU for at least 2 times and up to 6 times, with an average of 3.16 times.

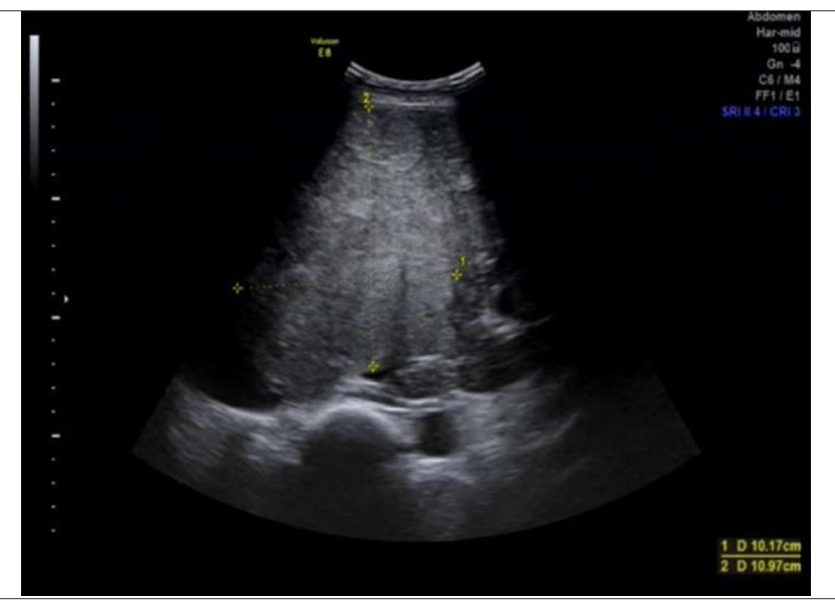

Figure 1: B-mode ultrasound image of patient.
After six months of treatment, the efficacy was evaluated according to WHO criteria, including complete remission, partial remission, stable and evolve. Complete remission revealed the arterial phase tumor focus basically disappears. Partial remission revealed arterial phase tumors shrink by more than $30 \%$ in diameter. Stable revealed the arterial phase tumor shrinks by no more than $30 \%$ or increases by no more than $20 \%$ in diameter. Evolve revealed the arterial phase focus increases by more than $20 \%$ in diameter. The total remission rate $=$ (complete remission + partial remission) / total number of cases $\times 100 \%$. After 6 months of treatment, fasting peripheral venous blood was collected from the two groups to measure and compare changes in indicators such as alpha-fetoprotein (AFP), alanine aminotransferase (ALT), aspartate amino-transferase (AST), and total bilirubin (TBIL). The incidence of complications at 6 months after surgery was observed in both groups. Both groups completed a 1-year follow-up survey to record recurrence and metastasis of the tumor in the form of telephone and outpatient review.

The SPSS 22.0 statistical software was used for data analysis. Count data was expressed as frequency with percentages and the Chi-square test was used for comparison between groups. Measurement data was expressed as mean \pm standard deviation, and independent samples t-test was used for comparison between groups. P-values less than or equal to 0.05 were considered significant.

\section{RESULTS}

Ninety patients included 52 males (57.78\%) and 38 females (42.22\%); age ranged from 35 to 73 years, and average age was $58.34 \pm 2.95$ years. Tumor diameter ranged from 4 to $15 \mathrm{~cm}$, with average of $11.16 \pm 3.28 \mathrm{~cm}$.

The total remission rate of the observation group was $84.44 \%$ (38 cases), which was higher than that of $62.22 \%$ ( 28 cases) in the control group $(p=0.017$, Table I). At 6 months after treatment, AFP level was lower in the observation group than that in the control group $(p<0.001)$. There was no statistical difference in liver function indicators of ALT, AST, and TBIL between the two groups $(p=0.968,0.944$ and 0.973 , respectively, Table II).

Table I: Comparison of treatment effects between the two groups.

\begin{tabular}{l|c|c|c|c|c|c|c}
\hline Groups & $\mathrm{n}$ & Complete remission & Partial remission & Stable & Evolve & Total remission rate & $\mathrm{p}$-value \\
\hline Control group & 45 & $6(13.33)$ & $22(48.89)$ & $11(24.44)$ & $6(13.33)$ & $28(62.22)$ & 0.017 \\
Observation group & 45 & $15(33.33)$ & $23(51.11)$ & $5(11.11)$ & $2(4.44)$ & $38(84.44)$ & \\
\hline
\end{tabular}

Table II: Comparison of AFP and liver function indicators in the two groups 6 months after treatment.

\begin{tabular}{|c|c|c|c|c|c|c|c|c|c|}
\hline \multirow[t]{2}{*}{ Groups } & \multirow[t]{2}{*}{$\mathrm{n}$} & \multicolumn{2}{|c|}{$\operatorname{AFP}(n g / m L)$} & \multicolumn{2}{|c|}{ ALT (U/L) } & \multicolumn{2}{|c|}{ AST (U/L) } & \multicolumn{2}{|c|}{ TBIL $(\mu \mathrm{mol} / \mathrm{L})$} \\
\hline & & Mean \pm SD & $\mathrm{p}$-value & Mean \pm SD & $\mathrm{p}$-value & Mean \pm SD & $\mathrm{p}$-value & Mean $\pm S D$ & $p$-value \\
\hline Control group & 45 & $112.32 \pm 10.02$ & $<0.001$ & $64.12 \pm 9.03$ & 0.968 & $55.96 \pm 11.92$ & 0.944 & $14.62 \pm 6.20$ & 0.973 \\
\hline Observation group & 45 & $85.47 \pm 7.15$ & & $64.05 \pm 7.35$ & & $56.13 \pm 10.96$ & & $14.66 \pm 4.77$ & \\
\hline
\end{tabular}


Table III: Comparison of complications between the two groups.

\begin{tabular}{|c|c|c|c|c|c|c|c|c|c|}
\hline Groups & $\mathrm{n}$ & $\begin{array}{c}\text { Digestive tract } \\
\text { hemorrhage }[\mathrm{n}(\%)]\end{array}$ & $p$-value & $\begin{array}{c}\text { Renal failure } \\
\text { [n(\%)] }\end{array}$ & $p$-value & $\begin{array}{l}\text { Fever } \\
{[\mathrm{n}(\%)]}\end{array}$ & $p$-value & $\begin{array}{c}\text { Nausea and } \\
\text { vomiting }[\mathrm{n}(\%)]\end{array}$ & $p$-value \\
\hline Control group & 45 & $6(13.33)$ & 0.049 & $0(0)$ & 0.315 & $4(8.89)$ & 0.694 & $5(11.11)$ & 0.725 \\
\hline Observation group & 45 & $1(2.22)$ & & $1(2.22)$ & & $3(6.67)$ & & $4(8.89)$ & \\
\hline
\end{tabular}

The incidence of digestive tract hemorrhage was $2.22 \%$ (1 case) in the observation group, which was lower than that of $13.33 \%$ ( 6 cases) in the control group $(p=0.049)$. There was no statistical difference in incidence of such complications as renal failure, fever, nausea and vomiting between the two groups $(p=0.315,0.694$ and 0.725 , respectively, Table III).

After one year of follow-up, the tumor recurrence rate in the observation group was $6.67 \%$ ( 3 cases), which is lower than that of $22.22 \%$ (10 cases) in the control group $(p=0.036)$. The tumor metastasis rate in the observation group was $4.44 \%$ (2 cases), which was lower than that of $17.78 \%$ ( 8 cases) in the control group $(p=0.044)$.

\section{DISCUSSION}

TACE is the most commonly used treatment for liver cancer. It is to embolize blood-supply hepatic arteries of liver cancer using embolic agents like lipiodol to induce ischemic necrosis of tumor. ${ }^{8,9}$ At the same time, use of chemotherapy drug can increase the efficacy of tumor destruction. Lee et al. concluded that the developed doxorubicin (DOX)-loaded, hyaluronic acid-ceramide (HACE) nanoassembly-releasing poly (lactic-co-glycolic acid) (PLGA) microspheres (MSs) can be used as a drug-loaded HA nanoassembly-releasing MS system for TACE therapy of liver cancer. ${ }^{10}$ Aktas et al. revealed that TACE, an important therapeutic option in unresectable liver tumors, can provide survival benefit even for intracellular cholangiocellular carcinoma and cancers of unknown primary, which are very large in size.11 However, liver cancer has dual blood supply from hepatic arterial and portal vein, and peripheral blood supply of large liver cancer comes from portal vein. Liver tumor cells are not sensitive to chemotherapy. ${ }^{12}$ Therefore, most studies suggest that it is difficult for TACE to completely kill liver tumor cells and improve the survival of middle and advanced liver cancer patients impossible to undergo resection. 13,14

HIFU is focused on the tumor target area through lowdensity ultrasound in vitro. The temperature of the focused point reaches $65-120^{\circ} \mathrm{C}$, so that the coagulative necrosis occurs in the tumor tissue, with almost no damage to the normal tissues around the tumor. ${ }^{15,16} \mathrm{At}$ the same time, as tumor antigens, the inactivated tumor tissue stimulates generation of anti-tumor antibodies in the body, creating subsequent immune anti-tumor effect. The effect in these two aspects enables fulfillment of the treatment purpose like a non-invasive surgical knife. ${ }^{17}$ Guiding HIFU treatment, using elastic properties of ablated tissues, is gaining attention since it is non- invasive and can provide new information. ${ }^{18} \mathrm{Ng}$ et al. concluded that HIFU treatment was effective for unresectable hepatocellular carcinoma and patients had favourable survival outcome. ${ }^{19}$ It is worth mentioning that even though the local and systemic complication should be a concern. Li et al. pointed out that complications of HIFU therapy for patients with liver cancer include skin burns, pain, induced fever, mild impairment of liver function or renal function and local damage. ${ }^{20}$ Synergic effects take place in the HIFU combined with TACE, in which lipiodol may play an important role in the synergic effects.

This study showed that the total remission rate was higher in the observation group than in control group; incidence of digestive tract hemorrhage was lower in the observation group than in the control group. There was no statistical difference in incidence of complications such as renal failure, fever, nausea and vomiting between the two groups. After one year of follow-up, the tumor recurrence rate and metastasis rate were lower in the observation group than in control group. It suggests that HIFU plus TACE is significantly superior to TACE alone in the treatment of primary liver cancer, as it can control tumor recurrence and metastasis without increasing adverse reactions, thus safe and reliable. This conclusion is basically consistent with some scholars. ${ }^{21}$ This may be because combination of HIFU and TACE can complement each other's advantages with a synergistic anti-cancer effect in which hyperthermia facilitates penetration of chemotherapy drugs into cancer cells, and hyperthermia combined with chemotherapy promotes the tumor cell apoptosis.

AFP is considered to be the most ideal marker in the diagnosis and treatment of liver cancer, which has great practical value in diagnosing tumors, monitoring tumor recurrence and metastasis, judging efficacy and prognosis, etc.22 Rungsakulkij et al. suggested that serum AFP responses were significant prognostic factors for the surgical outcomes of HCC patients, and post-resection AFP levels could direct the management of HCC patients. ${ }^{23}$ This study showed that AFP level was significantly lower in the observation group than in the control group at 6 months after treatment. There was no significant difference in liver function indicators between the two groups. It suggests that combination of HIFU and TACE can effectively lower AFP level, with little damage to normal liver tissue of primary liver cancer patients.

\section{CONCLUSION}

Compared with TACE alone, treatment of primary liver cancer by HIFU plus TACE has a higher overall 
remission rate, causes little damage to normal liver tissue, can fully kill tumor cells and reduce postoperative local recurrence and metastasis rate with less adverse reactions, thus worthy of clinical promotion.

\section{REFERENCES}

1. Thistle JE, Petrick JL, Yang B, Bradley MC, Graubard BI, McGlynn KA. Domperidone use and risk of primary liver cancer in the clinical practice research datalink. Cancer Epidemiol 2018; 55:170-5.

2. Melkonian SC, Jim MA, Reilley B, Erdrich J, Berkowitz Z, Wiggins $\mathrm{CL}$, et al. Incidence of primary liver cancer in American Indians and Alaska Natives, US, 1999-2009. Cancer Causes Control 2018; 29:833-44.

3. Mirzaei M, Ghoncheh M, Pournamdar Z, Soheilipour F, Salehiniya $\mathrm{H}$. Incidence and trend of liver cancer in Iran. J Coll Physicians Surg Pak 2016; 26:306-9.

4. Xu YH, Xiao A, Yang J, Zhang ZX, Zhang GX. Assessment of lipiodol deposition and residual cancer for hepatocellular carcinoma after transcatheter arterial chemoembolization via iodine-based material decomposition images with spectral computed tomography imaging: A preliminary study. Iran $\mathrm{J}$ Radiol 2015; 12:e26009.

5. Chen $\mathrm{Y}$, Chen H, Shi J. Nanobiotechnology promotes noninvasive high-intensity focused ultrasound cancer surgery. Adv Healthc Mater 2015; 4:158-65.

6. Diana M, Schiraldi L, Liu YY, Memeo R, Mutter D, Pessaux P, et al. High intensity focused ultrasound (HIFU) applied to hepato-bilio-pancreatic and the digestive system - current state of the art and future perspectives. Hepatobiliary Surg Nutr 2016; 5:329-44.

7. Chen B, Chen J, Luo Q, Guo C. Effective strategy of the combination of high-intensity focused ultrasound and transarterial chemoembolization for improving outcome of unresectable and metastatic hepatoblastoma: A retrospective cohort study. Transl Oncol 2014; 7:788-94.

8. Piscaglia F, Ogasawara S. Patient selection for transarterial chemoembolization in hepatocellular carcinoma: Importance of benefit/risk assessment. Liver Cancer 2018; 7:104-19.

9. Schicho A, Pereira PL, Michalik K, Beyer LP, Stroszczynski C, Wiggermann P. Safety and efficacy of transarterial chemoembolization with degradable starch microspheres (DSMTACE) in the treatment of secondary liver malignancies. Onco Targets Ther 2018; 11:345-50.

10. Lee SY, Choi JW, Lee JY, Kim DD, Kim HC, Cho HJ. Hyaluronic acid/doxorubicin nanoassembly-releasing micro-spheres for the transarterial chemoembolization of a liver tumor. Drug Deliv 2018; 25:1472-83.

11. Aktas G, Kus T, Metin T, Kervancioglu S, Elboga U. Long-term survival with transarterial chemoembolization and radioembolization in a patient with cancers of unknown primary. Onco Targets Ther 2018; 11:1885-9.

12. Lund P, Schubert D, Niketeghad F, Schirmacher P. Autocrine inhibition of chemotherapy response in human liver tumor cells by insulin-like growth factor-II. Cancer Lett 2004; 206:85-96.

13. Xiong ZP, Huang F, Lu MH. Association between insulin-like growth factor-2 expression and prognosis after transcatheter arterial chemoembolization and octreotide in patients with hepatocellular carcinoma. Asian Pac J Cancer Prev 2012; 13: 3191-4.

14. Li H, Hu Y, Li N, Zhou Y. Liver fibrosis and five-year survival of hepatocellular cancer cases undergoing transcatheter arterial chemo embolization using small doses. Asian Pac J Cancer Prev 2012; 13:1589-93.

15. Sengupta S, Balla VK. A review on the use of magnetic fields and ultrasound for non-invasive cancer treatment. J Adv Res 2018; 14:97-111.

16. Suzuki R, Maruyama K. Development of ultrasound theranostics for cancer. Yakugaku Zasshi 2018; 138:919-22.

17. Daher S, Massarwa M, Benson AA, Khoury T. Current and future treatment of hepatocellular carcinoma: an updated comprehensive review. J Clin Transl Hepatol 2018; 6:69-78.

18. de Senneville BD, Moonen C, Ries M. MRI-Guided HIFU methods for the ablation of liver and renal cancers. Adv Exp Med Biol 2016; 880:43-63.

19. Ng KK, Poon RT, Chan SC, Chok KS, Cheung TT, Tung H, et al. High-intensity focused ultrasound for hepatocellular carcinoma: A single-center experience. Ann Surg 2011; 253:981-7.

20. Li JJ, Gu MF, Luo GY, Liu LZ, Zhang R, Xu GL. Complications of high intensity focused ultrasound for patients with hepatocellular carcinoma. Technol Cancer Res Treat 2009; 8:217-24.

21. Zhao J, Zhang H, Wei L, Xie S, Suo Z. Comparing the longterm efficacy of standard and combined minimally invasive procedures for unresectable HCC: a mixed treatment comparison. Oncotarget 2017; 8:15101-13.

22. Tzartzeva K, Singal AG. Testing for AFP in combination with ultrasound improves early liver cancer detection. Expert Rev Gastroenterol Hepatol 2018; 12:947-9.

23. Rungsakulkij N, Suragul W, Mingphruedhi S, Tangtawee $P$, Muangkaew P, Aeesoa S. Prognostic role of alpha-fetoprotein response after hepatocellular carcinoma resection. World $\mathrm{J}$ Clin Cases 2018; 6:110-20. 